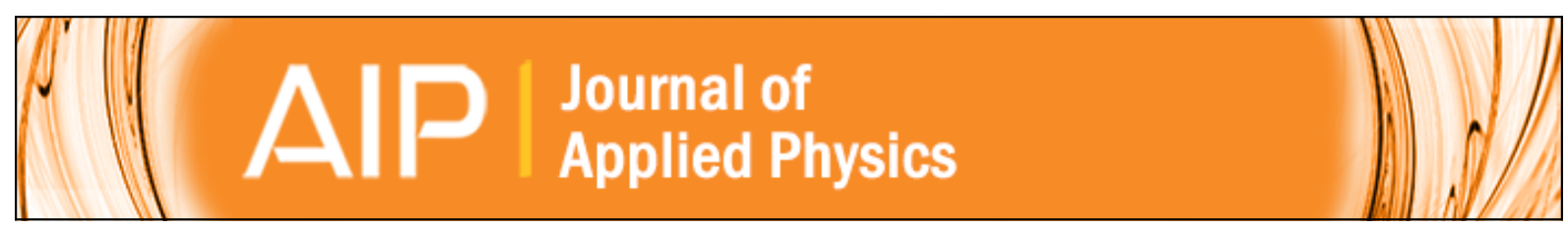

\title{
Remanence plots technique extended to exchange bias systems
}

A. Harres, R. Cichelero, L. G. Pereira, J. E. Schmidt, and J. Geshev

Citation: Journal of Applied Physics 114, 043902 (2013); doi: 10.1063/1.4816255

View online: http://dx.doi.org/10.1063/1.4816255

View Table of Contents: http://scitation.aip.org/content/aip/journal/jap/114/4?ver=pdfcov

Published by the AIP Publishing

Advertisement:

AlP Re-register for Table of Content Alerts

Create a profile.

Sign up today! 


\title{
Remanence plots technique extended to exchange bias systems
}

\author{
A. Harres, R. Cichelero, L. G. Pereira, J. E. Schmidt, and J. Geshev a) \\ Instituto de Física, Universidade Federal do Rio Grande do Sul (UFRGS), Porto Alegre, \\ 91501-970 Rio Grande do Sul, Brazil
}

(Received 3 June 2013; accepted 4 July 2013; published online 23 July 2013)

\begin{abstract}
Efforts have been recently made to use remanence plots in exchange-bias studies. However, since the two remnant magnetizations of a biased loop may differ, this technique cannot be applied in its classical form. This work extends it to systems with shifted loops and shows that the number of distinct plots is significantly increased. The approach was probed on Co/IrMn exchange bias films. Possible discrepancies between experiment and theory are pointed out and discussed. The adaptation of the model presented here enables it to become one of the few accessible techniques for rapid and accurate evaluation of magnetic interactions in biased systems. (C) 2013 AIP Publishing LLC.
\end{abstract} [http://dx.doi.org/10.1063/1.4816255]

\section{INTRODUCTION}

The exchange bias (EB) phenomenon, ${ }^{1}$ i.e., a shift of the hysteresis loop along the magnetic field axis, results from the exchange coupling between a ferromagnet (FM) and partially uncompensated spins at the interface with an adjacent antiferromagnet (AF). Despite the intense research efforts, understanding its mechanisms has remained a challenging task and EB still continues to receive great deal of attention. Emerging fields, such as EB manifestation in multiferroic systems, where a purely magnetic bias could be manipulated by an electrical field, further fuel the research on EB. Other types of interactions could also result in shifted loops. It has been theoretically shown that when an AF is in contact with a metallic FM, non-uniform quantum spin fluctuations may induce uncompensated spins in the $\mathrm{AF}$ giving rise to a dipole field responsible for bias $^{2}$ and that dipolar interactions between FM and rotatable anisotropy grains could lead to athermal training. ${ }^{3}$

There exists a number of approaches used to extract information contained in the specific shape of experimental hysteresis loops, e.g., their Fourier decomposition ${ }^{4}$ or demagnetization curve fitting. ${ }^{5}$ Recent attempts have been made to estimate interactions in EB systems ${ }^{6-8}$ using the so-called field-dependent remanence curves, namely, the isothermal remanence magnetization (IRM) and dc demagnetization (DCD) ones. A differentiated IRM or DCD curve represents the switching-field distribution (SFD), i.e., the dispersion of the fields at which irreversible rotations occur. ${ }^{9,10}$ The remanence curves are very sensitive to small changes in the remanence produced by magnetic interactions. ${ }^{11,12}$ This method has been originally developed to characterize interactions in monophase uniaxial or cubic anisotropy systems ${ }^{9,13-15}$ with symmetric hysteresis loops, where the remnant magnetizations of both loop branches are equal, differing only in sign. In biased systems, however, these remnant magnetizations may differ significantly and the technique cannot be applied in its classical form.

${ }^{a)}$ Email: julian@if.ufrgs.br
In the present work, a procedure that permits the use of the remanence plots in biased systems is proposed. Frequently, biased loops are asymmetric so two pairs of IRM and DCD curves coexist making the number of distinct Henkel $^{14}$ and $\delta M$ plots $^{9}$ four times greater than that obtained classically. Here, another two types of remanence plots related to the asymmetry of the magnetization reversal are introduced. The amended method was probed on polycrystalline $\mathrm{IrMn} / \mathrm{Cu} / \mathrm{Co}$ films.

\section{MAGNETIZATION CURVES PARAMETERS}

A proper comprehension of the magnetization curve parameters of EB systems is essential for the discussions presented below. In the textbooks on magnetism, the coercivity (or coercive field), $H_{\mathrm{c}}$, is defined as the value of the applied magnetic field, $H$, at which the magnetization, $M$, is zero. In the case of a conventional monophase system, $H_{\mathrm{c}}$ represents the half-width at half-height of the hysteresis loop. In EB systems, the coercivity of the descending branch of a hysteresis loop, $H_{\mathrm{c} 1}$, does not have the same absolute value as that of the ascending branch, $H_{\mathrm{c} 2}$, and $H_{\mathrm{c}}$ is habitually defined as $H_{\mathrm{c}}=\frac{1}{2}\left(H_{\mathrm{c} 2}-H_{\mathrm{c} 1}\right)$. However, due to other typical characteristics of the EB systems, i.e., the asymmetric magnetization reversal, this definition does not necessarily lead to value of $H_{\mathrm{c}}$ equal to the half-width at half-height of the loop. A more general definition ${ }^{16}$ using the switching fields of the two branches of the loop, $H_{\mathrm{sw} 1}$ and $H_{\mathrm{sw} 2}$, results in $H_{\mathrm{c}}^{\prime}=\frac{1}{2}\left(H_{\mathrm{sw} 2}-H_{\mathrm{sw} 1}\right)$. In a hysteresis loop trace, the shift along the field axis represents the so-called exchange-bias field $H_{\mathrm{eb}}=\frac{1}{2}\left|H_{\mathrm{c} 1}+H_{\mathrm{c} 2}\right|$; in terms of switching fields it becomes $H_{\mathrm{eb}}^{\prime 2}=\frac{1}{2}\left|H_{\mathrm{sw} 1}+H_{\mathrm{sw} 2}\right|$. All these parameters are visualized in Fig. 1.

Remnant magnetization, or also magnetic remanence $M_{\mathrm{r}}$, is the magnetization when $H=0$; similar to $H_{\mathrm{c}}$, it could be defined as $M_{\mathrm{r}}=\frac{1}{2}\left(M_{\mathrm{r} 1}-M_{\mathrm{r} 2}\right)$, where $M_{\mathrm{r} 1}$ (positive) and $M_{\mathrm{r} 2}$ (negative) are the respective remnant magnetizations of the descending and ascending branches of a major hysteresis loop. For an unbiased FM, as a general rule $M_{\mathrm{r} 1}=-M_{\mathrm{r} 2}$. An important question is if the above definition is appropriate for $\mathrm{EB}$ systems where, due to the negative (for the case of 


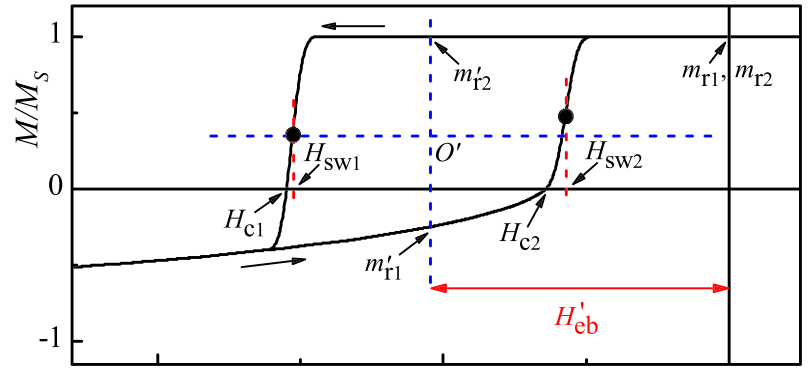

Magnetic field (arb. units)

FIG. 1. Scheme representing the parameters used in the characterization of exchange-bias systems. The arrows indicate the directions of the magnetic field sweeps.

conventional EB) shift along the field axis, $\left|M_{\mathrm{r} 2}\right|<M_{\mathrm{r} 1}$; in many cases $M_{\mathrm{r} 2}$ is positive or even equal to $M_{\mathrm{r} 1}$, as exemplified in Fig. 1, where the normalized (to the FM saturation magnetization $M_{\mathrm{s}}$ ) remnant magnetizations $m_{\mathrm{r} 1}=M_{\mathrm{r} 1} / M_{\mathrm{s}}$ and $m_{\mathrm{r} 2}=M_{\mathrm{r} 2} / M_{\mathrm{s}}$ are shown.

The remanence plots technique is based on a comparison of IRM and DCD curves and has been extensively used for estimation of interaction effects. ${ }^{9,11,12,15}$ The IRM curve, $m_{\mathrm{r}}(H)$, is measured on an initially demagnetized sample by applying a positive field $H_{i}$ which is then removed and $m_{\mathrm{r}}\left(H_{i}\right)$ is measured. Larger fields are then applied and the measurements repeated until positive saturation is reached. The DCD curve, $m_{\mathrm{d}}(H)$, is obtained by initially saturating the sample and then measuring $m_{\mathrm{d}}\left(H_{j}\right)$ after application of progressively larger reverse fields $H_{j}$. For the case of non-interacting uniaxial anisotropy monodomains in virgin or thermally-demagnetized samples, these curves are connected via the Wohlfarth's relation ${ }^{13} m_{\mathrm{d}}(H)=1-2 m_{\mathrm{r}}(H)$. Superparamagnetic, multidomain, and/or incoherent rotation effects could also lead to deviations from the linear relation. ${ }^{13} m_{\mathrm{d}}(H)$ versus $m_{\mathrm{r}}(H)$ is called Henkel plot ${ }^{14}$ which represents a straight line with a slope of -2 for the noninteracting case. Whereas the dependence of the interaction effects on the magnetic field is not plainly manifested in a Henkel plot, it is explicit in the so-called $\delta M$ plot, ${ }^{9}$

$$
\delta M(H)=2 m_{\mathrm{r}}(H)-1+m_{\mathrm{d}}(H) .
$$

The above relation should also be valid for acdemagnetized samples as long as the demagnetization is accomplished by using an infinite sequence of alternating field which amplitude decreases according to the chosen envelope function. In practice, however, only a finite sequence may be applied resulting in different levels of demagnetization. Zhang and Atherton ${ }^{17}$ showed that although the level of the demagnetized state may lead to different subsequent magnetization behaviors, the initial magnetization curves calculated through the Stoner-Wohlfarth model ${ }^{18}$ are practically identical for sufficiently high-level states. Hence, in such cases only, the remanence plots technique can be employed after ac demagnetization.

It is usually accepted that negative $\delta M$ values indicate "demagnetizing" interactions that tend to stabilize the demagnetized state; positive values are attributed to interactions promoting the magnetized state. Similarly, Henkel plots that lie above the straight line are typical of systems which are easier to magnetize than to demagnetize. This is true, however, for the uniaxial anisotropy case only. Noninteracting cubic anisotropy systems are characterized by positive $\delta M$ plots $^{15}$ and these may vary when anisotropies of different type co-exist. ${ }^{19}$

Since $m_{\mathrm{r}}(H)$ depends on the method by which the demagnetized state is produced, one can obtain very different $m_{\mathrm{r}}(H)$ after dc, ac, or thermal demagnetization and the corresponding remanence plots can vary significantly. For the case of uniaxial anisotropy, Bissel et al. ${ }^{11}$ have derived a connection between $m_{\mathrm{d}}(H)$ and the IRM curve after dc demagnetization, $m_{\mathrm{r}}^{\mathrm{dc}}(H)$, produced by cycling the sample to the negative remanence coercivity $\left(-H_{\mathrm{r}}\right)$ after previous saturation, and then reducing $H$ to zero. The resulting connections are $m_{\mathrm{r}}^{\mathrm{dc}}(H)=1-m_{\mathrm{d}}(H)$ for $H<H_{\mathrm{r}}$ and $m_{\mathrm{r}}^{\mathrm{dc}}(H)=1$ for $H \geq H_{\mathrm{r}}$. The $\delta M^{\mathrm{dc}}$ plot has been defined ${ }^{20}$ as

$$
\delta M^{\mathrm{dc}}(H)=m_{\mathrm{r}}^{\mathrm{dc}}(H)-1+m_{\mathrm{d}}(H)
$$

for $H<H_{\mathrm{r}}$, and

$$
\delta M^{\mathrm{dc}}(H)=m_{\mathrm{r}}^{\mathrm{dc}}(H)-1
$$

for $H \geq H_{\mathrm{r}}$. In case of no interactions and uniaxial anisotropy, $\delta M^{\mathrm{dc}}=0$ for all values of $H$; the corresponding Henkel plot is a straight line with a slope of -1 . The variations of these plots with the demagnetized state of $m_{\mathrm{r}}$ have also been demonstrated via model simulations ${ }^{21,22}$ and it has been emphasized that the demagnetization method must be specified if any conclusions are to be drawn from a remanence plot. Undoubtedly, this technique could only be applied on magnetic systems that can be repeatedly demagnetized in a reliable and reproducible manner, which is the case of the films studied here.

As stressed above, in EB systems $m_{\mathrm{r} 1}+m_{\mathrm{r} 2} \neq 0$ making the above method impracticable. Here, we propose that the reference frame of $M(H)$ should be changed in a manner that the new origin, $O^{\prime}$, coincides with the center of the original shifted loop as exemplified in Fig. 1. The new remnant magnetizations are normalized to $m_{\mathrm{eb}}=\frac{1}{2}\left(m_{\mathrm{eb}}^{\text {up }}-m_{\mathrm{eb}}^{\text {down }}\right)$, being $m_{\mathrm{eb}}^{\mathrm{up}}$ and $m_{\mathrm{eb}}^{\text {down }}$ the normalized magnetizations at $H_{\mathrm{eb}}$ of the descending and ascending branches of the major loop, respectively. Performed the above operation, the remanence plots technique can be readily applied.

Recall, however, that the majority of EB hysteresis loops are intrinsically asymmetric, which is reflected on the respective $m_{\mathrm{r}}(H)$ and $m_{\mathrm{d}}(H)$. There can be traced two IRM curves, $m_{\mathrm{r}}^{+}$and $m_{\mathrm{r}}^{-}$, for each method (ac or dc) used to achieve $m_{\mathrm{r}}=0$, and two DCD ones, namely, $m_{\mathrm{d}}^{+}$and $m_{\mathrm{d}}^{-}$, greatly increasing the number of $\delta M$ and Henkel plots that can be built. Here, "+" or "_" refers to the field sweep direction of the remanence curve. It seems intuitive that "+" ("-") in $m_{\mathrm{d}}^{+}\left(m_{\mathrm{d}}^{-}\right)$also indicates that the sign of the saturating magnetic field last applied before start achieving this curve is negative (positive). "+" ("-") in $m_{\mathrm{r}}(H)$ notation, however, implies that the saturating field used before demagnetization was positive (negative) as it is in the classical procedure ${ }^{13}$ to achieve $m_{\mathrm{r}}(H)$. 
Here, we introduce another two types of plots,

$$
\begin{aligned}
& \Delta m_{\mathrm{r}}(H)=m_{\mathrm{r}}^{-}(H)-m_{\mathrm{r}}^{+}(H), \\
& \Delta m_{\mathrm{d}}(H)=m_{\mathrm{d}}^{-}(H)-m_{\mathrm{d}}^{+}(H) .
\end{aligned}
$$

As shown below, these plots can also be used to characterize the magnetization reversal mechanisms and/or interactions in systems with asymmetric hysteresis loops.

\section{RESULTS AND DISCUSSION}

This approach was tested on a polycrystalline FM/AF (Co/IrMn) system, namely, a series of films composed by $\mathrm{Ta}(5 \mathrm{~nm}) / \mathrm{Ru}(15 \mathrm{~nm}) / \mathrm{Co}(5 \mathrm{~nm}) / \mathrm{Cu} / \mathrm{Ir}_{20} \mathrm{Mn}_{80}(7 \mathrm{~nm}) / \mathrm{Ta}(3 \mathrm{~nm})$, for $\mathrm{Cu}$ layer thickness $t_{\mathrm{Cu}}$ varied between 0 and $2.0 \mathrm{~nm}$, deposited onto $\mathrm{Si}(100)$ substrates via magnetron sputtering (base pressure of $1.4 \times 10^{-7}$ Torr, Ar pressure of $2 \mathrm{mTorr}$ for the deposition of $\mathrm{Ru}, \mathrm{Ta}, \mathrm{Cu}$ and $\mathrm{Co}$, and 7.5 mTorr for IrMn). A film containing only the FM, i.e., Ta $(5 \mathrm{~nm}) /$ $\mathrm{Ru}(15 \mathrm{~nm}) / \mathrm{Co}(5 \mathrm{~nm}) / \mathrm{Cu}(3 \mathrm{~nm})$, has also been deposited. To set the EB, the films were grown in in-plane magnetic field of 180 Oe. All magnetic measurements were made at room temperature via an alternating gradient-field magnetometer with in-plane $\mathbf{H}$ applied along the growth-induced EB direction. None of the samples showed appreciable training effect, i.e., a variation of the hysteresis loop's characteristics upon subsequent field cycling, see Ref. 3 and references therein.

A pair of $m_{\mathrm{r}}^{\mathrm{dc}}(H)$ and $m_{\mathrm{d}}(H)$ as well as $\delta M_{\mathrm{dc}}$ and Henkel plots $\left(h p_{\mathrm{dc}}\right)$ for the film with $t_{\mathrm{Cu}}=0$ are shown in Figs. 2 and 3, respectively. The signs in the "++," "+-," "-+," or "- _" notations refer to those of the respective $m_{\mathrm{r}}(H)$ or $m_{\mathrm{d}}(H)$. For example, in $\delta M_{\mathrm{dc}}^{-+}(H)$, equal to $m_{\mathrm{r}}^{-\mathrm{dc}}(H)-1$ $+m_{\mathrm{d}}^{+}(H)$ for $H<H_{\mathrm{r}}$ and $m_{\mathrm{r}}^{-\mathrm{dc}}(H)-1$ otherwise, zero remanence is attained by dc demagnetization after negative saturation; $m_{\mathrm{d}}(H)$ is measured after negative saturation as well.

For better visualization, when a $\delta M$ plot with mixed signs ("-+" or "+-") is constructed, the field sweep direction of $m_{\mathrm{d}}(H)$ is reversed. Such $\delta M$ plots show only positive (for "-+" pairs) or negative (for "+-" ones) values. The dc "-+" Henkel plot, $h p_{\mathrm{dc}}^{-+}$, is almost rectangular owing to the fact that $m_{\mathrm{d}}$ used varies very little in the field range where $m_{\mathrm{r}}$ is practically reversed, see $m_{\mathrm{r}}^{-}(H)$ and $m_{\mathrm{d}}^{+}(H)$ and the SFDs plotted in Fig. 2; this, to a certain extent, holds for the rest of

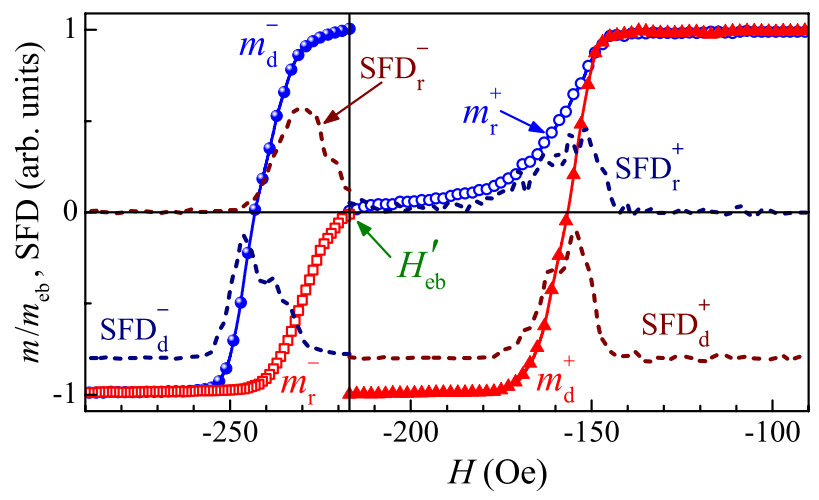

FIG. 2. Remanence curves for the Co/IrMn film, where $m_{\mathrm{r}}=0$ was attained by de demagnetization; dashed lines: the respective SFDs. The solid lines are guides to the eyes.
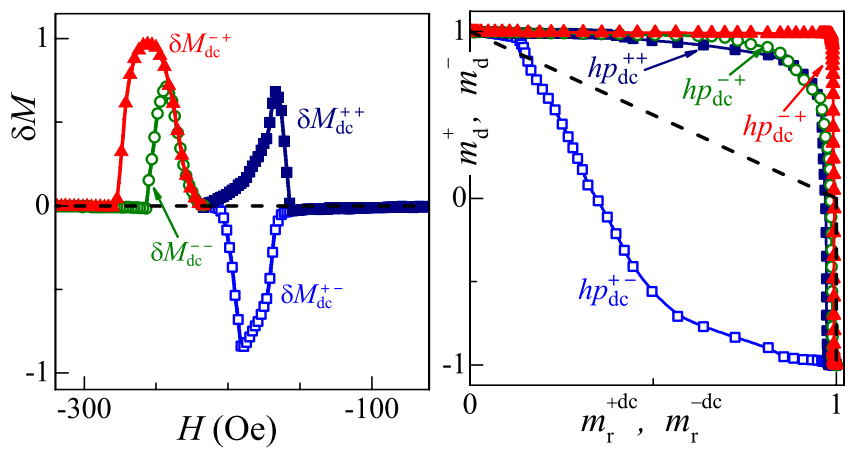

FIG. 3. $\delta M$ (left) and Henkel (right) plots for the Co/IrMn film obtained after dc demagnetization performed before obtaining $m_{\mathrm{r}}(H)$. The solid ones are guides to the eyes and the dashed lines correspond to the unbiased noninteracting uniaxial anisotropy case.

the Henkel plots in Fig. 3. In other words, their rather extreme shapes come from the high asymmetry between the magnetization reversals that take place for descending and ascending field sweeps, which is one of the intrinsic EB characteristics.

Figure 4 shows $h p_{\mathrm{dc}}^{--}$of the Co single layer and representative plots obtained for the $\mathrm{Co} / \mathrm{Cu}\left(t_{\mathrm{Cu}}\right) / \mathrm{IrMn}$ films. The plots of the FM film and of the FM/AF sample with rather thick $\mathrm{Cu}$ spacer of $1 \mathrm{~nm}$ (which practically decouples the FM and AF layers) are very similar, both lying very close to the non-interacting line. The FM/AF plot presents the more pronounced positive deviation, which turns to be less prominent with the increase in $t_{\mathrm{Cu}}$.

Henkel plots for different FM/AF ferromagnetic coupling strengths, simulated through a model based on the Landau-Lifshitz-Gilbert equation of magnetization motion, are plotted in the right panel of Fig. 4. Let briefly sketch the model that considers a polycrystalline FM/AF thin film consisting of an ensemble of grains. Each of these grains is treated as a magnetic monodomain with distinct anisotropy and coupling parameters. The monodomains are organized in layers that form a $3 \mathrm{D}$ grid with uniform $32 \times 32 \times 4$ mesh spacing; the nodes interact ferromagnetically with their nearest neighbors. It is assumed that the spins at the AF part of
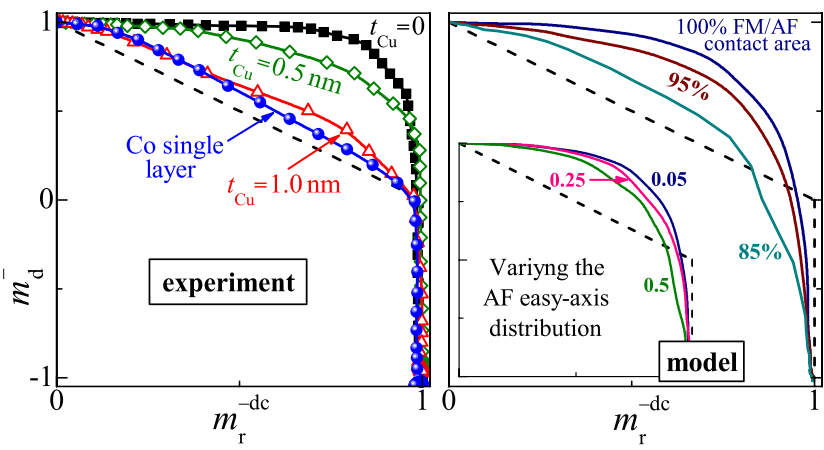

FIG. 4. Left: representative experimental Henkel plots for the Co single layer and for the $\mathrm{Co} / \mathrm{Cu}\left(t_{\mathrm{Cu}}\right) / \mathrm{IrMn}$ films obtained after dc demagnetization. Right: Plots simulated for different percentages of the FM/AF contact area (using as parameters $\sigma_{\varphi}^{\mathrm{FM}}=1.3 \mathrm{rad}, \sigma_{\varphi}^{\mathrm{AF}}=0.5 \mathrm{rad}, H_{\mathrm{E}}^{\mathrm{FM} / \mathrm{FM}} / M_{s}=15$, $H_{\mathrm{E}}^{\mathrm{FM} / \mathrm{AF}} / M_{s}=45$, and $H_{\mathrm{U}}^{\mathrm{FM}} / M_{s}=130$ as a mean value with respective $\sigma_{\mathrm{U}}^{\mathrm{FM}} / M_{s}=0.28$. The inset shows plots simulated as the one with $100 \% \mathrm{FM} /$ $\mathrm{AF}$ contact area for three different $\sigma_{\varphi}^{\mathrm{AF}}$ (in radians). The dashed lines correspond to the non-interacting case and the solid lines are guides to the eyes. 
the interface have anisotropy sufficiently strong so their initial orientations remain practically unchanged, providing an uncompensated FM/AF interface. The values of the uniaxial FM magnetic anisotropy follow a log-normal distribution characterized by $\sigma_{\mathrm{U}}^{\mathrm{FM}}$; the directions of in-plane easy axes obey a Gaussian distribution with standard deviation $\sigma_{\varphi}^{\mathrm{FM}}$ (or $\left.\sigma_{\varphi}^{\mathrm{AF}}\right)$. The effective field acting on each element is a sum of the external, uniaxial anisotropy $\left(H_{\mathrm{U}}^{\mathrm{FM}}\right.$ or $\left.H_{\mathrm{U}}^{\mathrm{AF}}\right)$, and exchange-coupling $\left(H_{\mathrm{E}}^{\mathrm{FM} / \mathrm{FM}}, H_{\mathrm{E}}^{\mathrm{AF} / \mathrm{AF}}\right.$, and/or $\left.H_{\mathrm{E}}^{\mathrm{FM} / \mathrm{AF}}\right)$, fields.

Henkel plots, simulated for different percentages of the $\mathrm{FM} / \mathrm{AF}$ contact interfacial area (which is proportional to the effective FM/AF exchange coupling and, consequently, to the EB), are shown in Fig. 4. A clear trend of decreasing the positive deviations with the decrease in the FM/AF coupling area is seen. This clearly indicates that the interface coupling in biased systems can, at least qualitatively, be estimated with the help of the amended remanence plots technique. Moreover, the simulation results given in the inset of this figure demonstrate that this method could also give information of how the easy-axis distribution of the uncompensated interface spins changes with, e.g., the growth or post-depositiontreatment parameters of the sample.

The "++" plots in Fig. 3 show positive and weak negative (better visualized in the region where $m_{\mathrm{d}}<0$ ) deviations from the lines corresponding to the non-interacting case, also seen in $h p_{\text {ac }}^{++}$(not shown here). This might indicate coexistence of magnetizing (exchange) and demagnetizing (e.g., dipolar) interactions if the traditional remanence plots interpretation is used. Calculations via slightly-modified version of the model sketched above assuming antiferromagnetic exchange interactions within the AF layer show that positive and negative deviations could be obtained simultaneously in a Henkel plot considering exchange coupling only, indicating that the interpretation of remanence plots of biased systems is not necessarily the conventional one. Certainly, more systematic research needs to be conducted to further clarify these issues, which is the aim of forthcoming studies.

The here-defined $\Delta m_{\mathrm{r}}$ and $\Delta m_{\mathrm{d}}$ plots for the FM/AF film with $t_{\mathrm{Cu}}=0$ are shown in Fig. 5(a). These, together with the rest of the $\delta M$ plots, could be used to estimate interaction effects and asymmetry of the magnetization reversal (for samples with biased though symmetric loops, each of these plots will be essentially zero) as exemplified below. $\Delta m_{\mathrm{d}}$

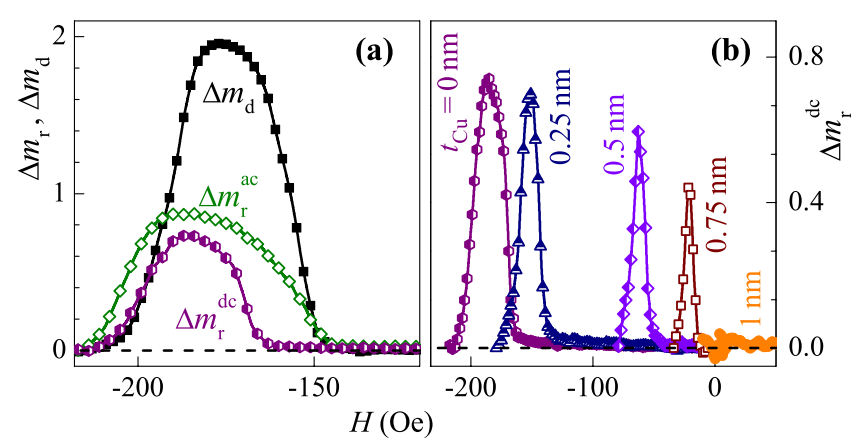

FIG. 5. (a) The here-defined $\Delta m_{\mathrm{r}}$ and $\Delta m_{\mathrm{d}}$ plots for the FM/AF film $\left(t_{\mathrm{Cu}}=0\right)$ and (b) $\Delta m_{\mathrm{r}}^{\mathrm{dc}}$ as a function of $t_{\mathrm{Cu}}$ for the $\mathrm{Co} / \mathrm{Cu} / \mathrm{IrMn}$ series. The solid lines are guides to the eyes. plots, in particular, are much easier (and faster) to acquire than any other plot given that there is no need to demagnetize the sample.

Figure 5(b) gives $\Delta m_{\mathrm{r}}^{\mathrm{dc}}$ for the films with $t_{\mathrm{Cu}} \leq 1.0 \mathrm{~nm}$ (the plots for higher $t_{\mathrm{Cu}}$ are practically identical to that of $\left.t_{\mathrm{Cu}}=1.0 \mathrm{~nm}\right)$. The maximum value of $\Delta m_{\mathrm{r}}^{\mathrm{dc}}$ decreases and its position shifts towards low-field values when $t_{\mathrm{Cu}}$ is increased confirming that the effective FM/AF exchange coupling decreases as the thickness of the spacer is increased. $^{23,24}$ The same holds for the asymmetries in the hysteresis loop's shape and in the magnetization reversal, i.e., $\Delta m_{\mathrm{r}}^{\mathrm{dc}}$ versus $t_{\mathrm{Cu}}$ indicates that the FM is practically uncoupled for $t_{\mathrm{Cu}} \geq 1.0 \mathrm{~nm}$.

The experimental and model results presented in Figs. 4 and 5 demonstrate that the modified remanence curves method can be applied to exchange-biased systems allowing not only for qualitative interface coupling assessment but also for estimation of the particular (AF and/or FM) easy-axis distributions. Quantitative evaluation of the interactions could further be attained with the help of appropriate model calculations.

In summary, the remanence plots technique was extended to systems with shifted hysteresis loops where the number of distinct remanence curves is significantly increased; another two types of remanence plots related to the asymmetry of the magnetization reversal were also introduced. The amended method was applied to a polycrystalline $\mathrm{Co} / \mathrm{IrMn}$ exchange-bias films. Plots of different types were probed and deviations from the theory were pointed out and discussed. After the adaptation, the method has the potential to become an accessible technique for rapid and accurate evaluation of magnetic interactions in biased systems.

\section{ACKNOWLEDGMENTS}

The authors appreciate useful discussions with V. Skumryev and D. Givord. The sputtering deposition was performed at the Laboratório de Conformação Nanométrica at Instituto de Física, UFRGS. This work has been supported by the Brazilian agencies CNPq (Project Nos. 304543/20098, 506323/2010-2, 141797/2011-8, and 483277/2012-6) and FAPERGS (Project No. 12/2778-4).

${ }^{1}$ W. H. Meiklejohn and C. P. Bean, Phys. Rev. 102, 1413 (1956); 105, 904 (1957).

${ }^{2}$ G. J. Mata, E. Pestana, H. Dreysse, and M. Kiwi, Phys. B: Condens. Matter 398, 262 (2007).

${ }^{3}$ A. Harres and J. Geshev, J. Phys.: Condens. Matter 23, 216003 (2011).

${ }^{4}$ M. Jackson, H.-U. Worm, and S. K. Banerjee, Phys. Earth Planet. Inter. 65, 78 (1990); V. Masheva, J. Geshev, and M. Mikhov, J. Magn. Magn. Mater. 137, 350 (1994).

${ }^{5}$ K.-D. Durst and H. Kronmüller, J. Magn. Magn. Mater. 59, 86 (1986); V. Masheva, M. Grigorova, N. Valkov, H. J. Blythe, T. Midlarz, V. Blaskov, J. Geshev, and M. Mikhov, ibid. 196-197, 128 (1999).

${ }^{6}$ H. W. Zhang, C. B. Rong, X. B. Du, J. Zhang, S. Y. Zhang, and B. G. Shen, Appl. Phys. Lett. 82, 4098 (2003).

${ }^{7}$ N. Domingo, D. Fiorani, A. M. Testa, C. Binns, S. Baker, and J. Tejada, J. Phys. D: Appl. Phys. 41, 134009 (2008).

${ }^{8}$ N. Lakshmi, H. Bhargava, O. P. Suwalka, K. Venugopalan, V. Sebastian, V. R. Reddy, and A. Gupta, Phys. Rev. B 80, 174425 (2009).

${ }^{9}$ P. E. Kelly, K. O'Grady, P. I. Mayo, and R. W. Chantrell, IEEE Trans. Magn. 25, 3881 (1989).

${ }^{10}$ R. W. Chantrell and K. O’Grady, J. Phys. D: Appl. Phys. 25, 1 (1992). 
${ }^{11}$ P. Bissel, R. Chantrell, G. Tomka, J. Knowles, and M. Sharrock, IEEE Trans. Magn. 25, 3650 (1989).

${ }^{12}$ P. I. Mayo, K. G'Grady, R. W. Chantrell, J. A. Cambridge, I. L. Sanders, T. Yogi, and J. K. Howard, J. Magn. Magn. Mater. 95, 109 (1991).

${ }^{13}$ E. P. Wohlfarth, J. Appl. Phys. 29, 595 (1958).

${ }^{14}$ O. Henkel, Phys. Status Solidi 7, 919 (1964).

${ }^{15}$ J. Geshev and M. Mikhov, J. Magn. Magn. Mater. 104-107, 1569 (1992).

${ }^{16}$ L. Sun, P. C. Searson, and C. L. Chien, Phys. Rev. B 71, 012417 (2005).

${ }^{17}$ Y. Zhang and D. L. Atherton, IEEE Trans. Magn. 31, 2233 (1995).

${ }^{18}$ E. C. Stoner and E. P. Wohlfarth, Philos. Trans. R. Soc. London Ser. A 240, 599 (1948).

${ }^{19}$ J. Geshev, M. Mikhov, and J. E. Schmidt, J. Appl. Phys. 85, 7321 (1999).
${ }^{20}$ A. D. C. Viegas, J. Geshev, L. S. Dorneles, J. E. Schmidt, and M. Knobel, J. Appl. Phys. 82, 3047 (1997).

${ }^{21}$ F. Vajda, E. Della Torre, and R. D. McMichael, J. Appl. Phys. 75, 5689 (1994).

${ }^{22}$ R. D. McMichael, F. Vajda, and E. Della Torre, J. Appl. Phys. 75, 5692 (1994).

${ }^{23}$ J. Geshev, S. Nicolodi, L. G. Pereira, L. C. C. M. Nagamine, J. E. Schmidt, C. Deranlot, F. Petroff, R. L. Rodríguez-Suárez, and A. Azevedo, Phys. Rev. B 75, 214402 (2007).

${ }^{24}$ S. Nicolodi, A. Harres, L. G. Pereira, J. E. Schmidt, M. A. de Sousa, F. Pelegrini, A. D. C. Viegas, C. Deranlot, F. Petroff, and J. Geshev, J. Appl. Phys. 110, 063922 (2011). 